\title{
Global Analysis of COVID-19 Clinical Related Trials
}

\author{
Muthusami Rathinasamy, Saritha Kandhasamy \\ ${ }^{1}$ Department of Computer Applications, Dr. Mahalingam College of Engineering and Technology, Anna University, \\ Tamil Nadu, India \\ ${ }^{2}$ Department of Mathematics, P.A. College of Engineering and Technology, Anna University, Tamil Nadu, India \\ These two authors contributed equally to this article
}

\begin{abstract}
Objectives: The aim of this study was to analyze clinical trials using topic modelling to identify emerging vaccine progression topics around the globe.

Methods: The most important computational problem for the modeling of topics is using the observed documents to infer the hidden topic structure. The topic model was developed on the basis of data recorded on the WHO International Clinical Trials Registry Platform (ICTRP) between 3 February and 11 August 2020.

Results: Show the top 10 topics based on the topic model that has been created. These topics are explored in the reviews and studies of clinical trials related to the development of the COVID-19 vaccine.

Conclusion: This study aimed at the clinical trials registered at ICTRP and showed the recorded monthly clinical trials as well as the top 15 countries vying for the COVID-19 vaccine from 3 February to 11 August 2020. Using the dataset of the collected clinical trials, the topic model was developed to examine emerging trends in vaccine progression. J Microbiol Infect Dis 2020; 10(4):xxxxx.
\end{abstract}

Keywords: COVID-19, Clinical trials, Vaccine progression, Emerging topics, Topic model

\section{INTRODUCTION}

Numerous pneumonia cases were identified due to a mystery virus in December 2019, in Wuhan, Hubei, China. In addition, the World Health Organization (WHO) had issued an epidemiological warning dated 31 December 2019. The Chinese scientists had identified severe acute respiratory syndrome coronavirus 2 (SARSCoV-2) as the causative pathogen [1]. SARS-CoV-2, the cause of coronavirus disease 2019 (COVID-19), has disseminated all over the globe leading to the ongoing pandemic. COVID19 had inflicted $21,294,845$ patients and resulted in 761,779 deaths as of 16 August 2020 as stated in the daily situation report of the WHO [2]. In this situation, medicine domain scientists, and researchers from over 150 countries are trying to find treatments and medicines that can save the lives of infected people [3]. As of 20 August 2020, researchers around the world had constructed more than 165 coronavirus vaccine candidates and 32 of them are in clinical trials [4]. Currently, COVID-19 has no effective treatment. Therefore, it is of interest to develop treatment and prevention agents to combat the viral outbreak. We are specifically interested in gleaning information using clinical trial data available in the public domain to foresee vaccine projections.

A clinical trial would be any scientific study that retrospectively applies one or more healthrelated treatments to particular people or groups of people to determine the impact on health outcomes [5-7]. Even clinical trials can be called interventional trials. Interventions include, but are not limited to, medications, enzymes, as well as other biologicals, medical interventions, radiological procedures, devices, behavioral treatments, changes in the treatment process, preventative medicine, etc. It includes Phase I to Phase IV trials in this conceptualization. The International Clinical Trials Registry Platform

Correspondence: Dr. Muthusami Rathinasamy, Department of Computer Applications, Dr. Mahalingam College of Engineering and Technology, Anna University, Tamil Nadu, India 
(ICTRP) is a multinational program aimed at making knowledge freely accessible about all clinical trials involving humans. If an authority in a member State (such as a Ministry of Health or a National Regulatory Authority) is interested in creating a new registry of clinical trials that fulfills WHO requirements (or improving an existing registry), or in developing policies that involve registration in an existing primary registry, then they may approach the ICTRP for assistance [8].

\section{METHODS}

\section{Dataset}

The clinical trials dataset was collected from the International Clinical Trials Registry Platform (ICTRP) of the WHO which was registered between 3 February and 11 August 2020. It contains 4957 records with 43 fields including Trial ID, Last Refreshed on, Public title, Primary sponsor, Date registration, age details, Study type, Phase, Countries, Contact details, results details. In this study, these records were used to analyze emerging vaccine progression topics across the globe between 3 February and 11 August 2020.

\section{Analysis}

To construct the topic model, the document-term matrix (DTM) was computed using the field of public title, which is the textual content of clinical trials. The purpose of the topic modeling is an eventual discovery of the topics in a collection of documents. The structure of the topic is the distribution of topics per document, and words per topic, i.e. hidden structure. The most important computational problem for the modeling of topics is using the observed documents to infer the hidden topic structure. The topic model such as latent Dirichlet allocation (LDA) [9] was implemented on dataset in this work. The number of topics should be pre-set in advance, in order to fit the LDA model to a specific document-term matrix. Moreover, the estimation, using Gibbs sampling, requires value information for earlier distribution parameters. The following have been set in our experimental work; $10 / \mathrm{k}$ for $\alpha$, the topic distribution of documents $\theta$ and 0.1 for $\phi$, and the term distribution of the topics $\beta$.
All experiments were conducted on a machine with the following specifications: Processor: Intel Core i3 @ 2.20 GHz, RAM: 8 GB, Windows 8; 64-bit operating system and $\mathrm{R}$ version 3.5.1.

\section{RESULTS}

Figure 1 shows the number of trials registered between February 3 and August 11, 2020. In the month of February 186 clinical trials were reported followed by $416,453,844,950$ and 1223 in the months of March, April, June and July respectively. As noted, the clinical trials for COVID-19 are gradually increasing with 884 clinical trials recorded in August.

Figure 2 displays the top 15 countries worldwide based on the number of clinical trials registered at ICTRP as of 11 August 2020. It shows that China is in top with 855 clinical trials, others with 726, 512, 444, 336, 293, 291 236, 126, 126, 118, 115, 103, 101 and 94 trials are USA, France, Iran, India, Germany, Spain, Italy, Brazil, Canada, the Netherlands, Egypt, Japan, Australia and Belgium.

The visualization in Figure 3 helps us understand the 10 topics related to COVID-19 vaccine progression which is derived from the topic model that has been created. The topic \#1 narrated the clinical characteristics of pneumonia coronavirus patients, the topic \#2 reported as randomized, double blind placebocontrolled trial to evaluate and safety of patients, the topic \#3 stated as prospective cohort study of convalescent plasma, the topic \#4 indicated that COVID-19 acute respiratory distress syndrome (ARDS), the topic \#5 says that study of immune response to SARS infection, the topic \#6 described the management of critically ill patients with pulmonary, the topic \#7 labelled the multicenter, open-label, randomizedcontrolled trial, the topic \#8 portrayed the psychological impact of the COVID-19 pandemic on healthcare workers, the topic \#9 described the link between patients vitamin levels with risk factors for COVID-19 and the topic \#10 tells that hydroxychloroquine for the treatment of patients with mild to moderate COVID-19. 


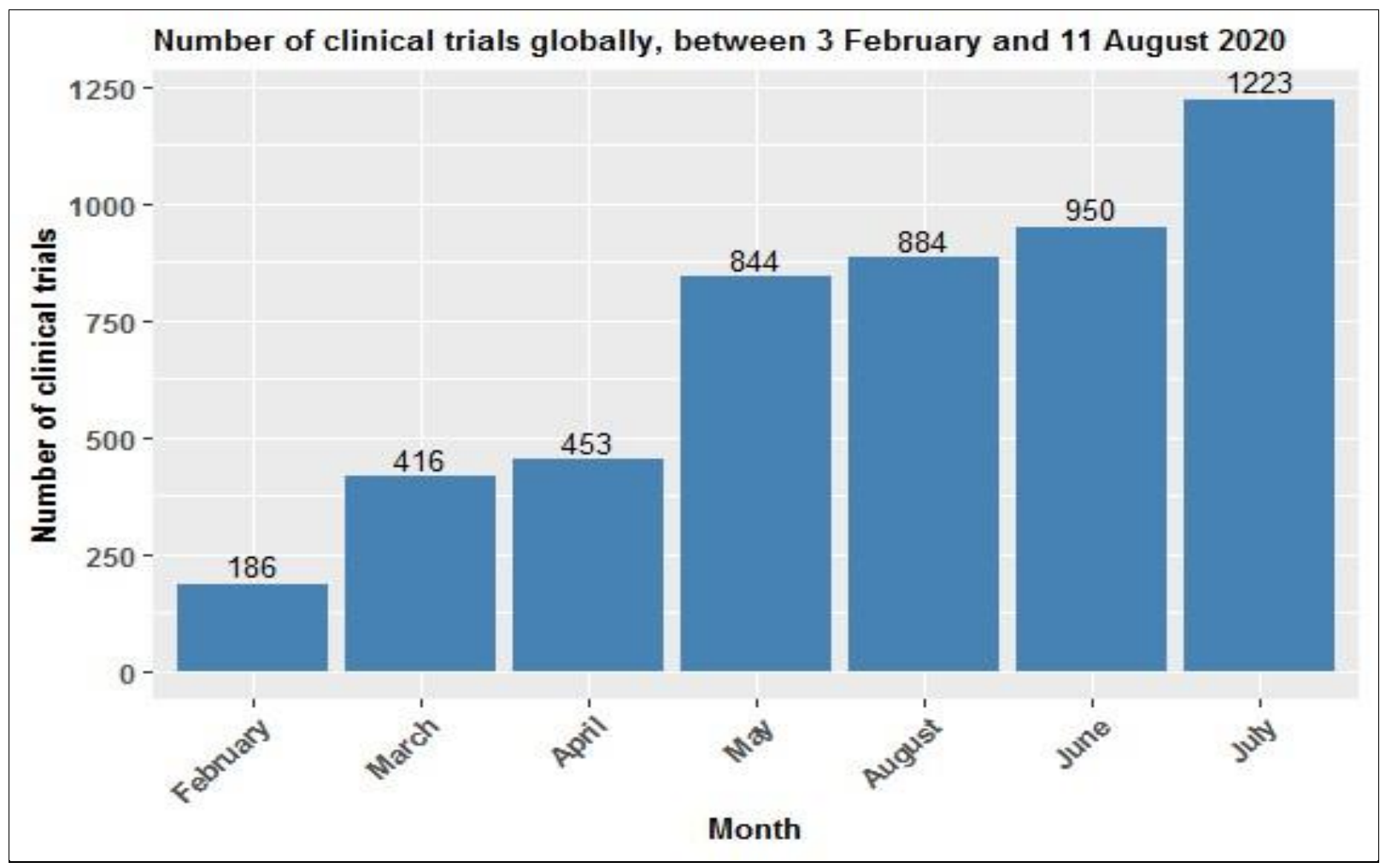

Figure 1. Showing the monthly wise clinical trials between 3 February and 11 August 2020 registered at the International Clinical Trials Registry Platform.

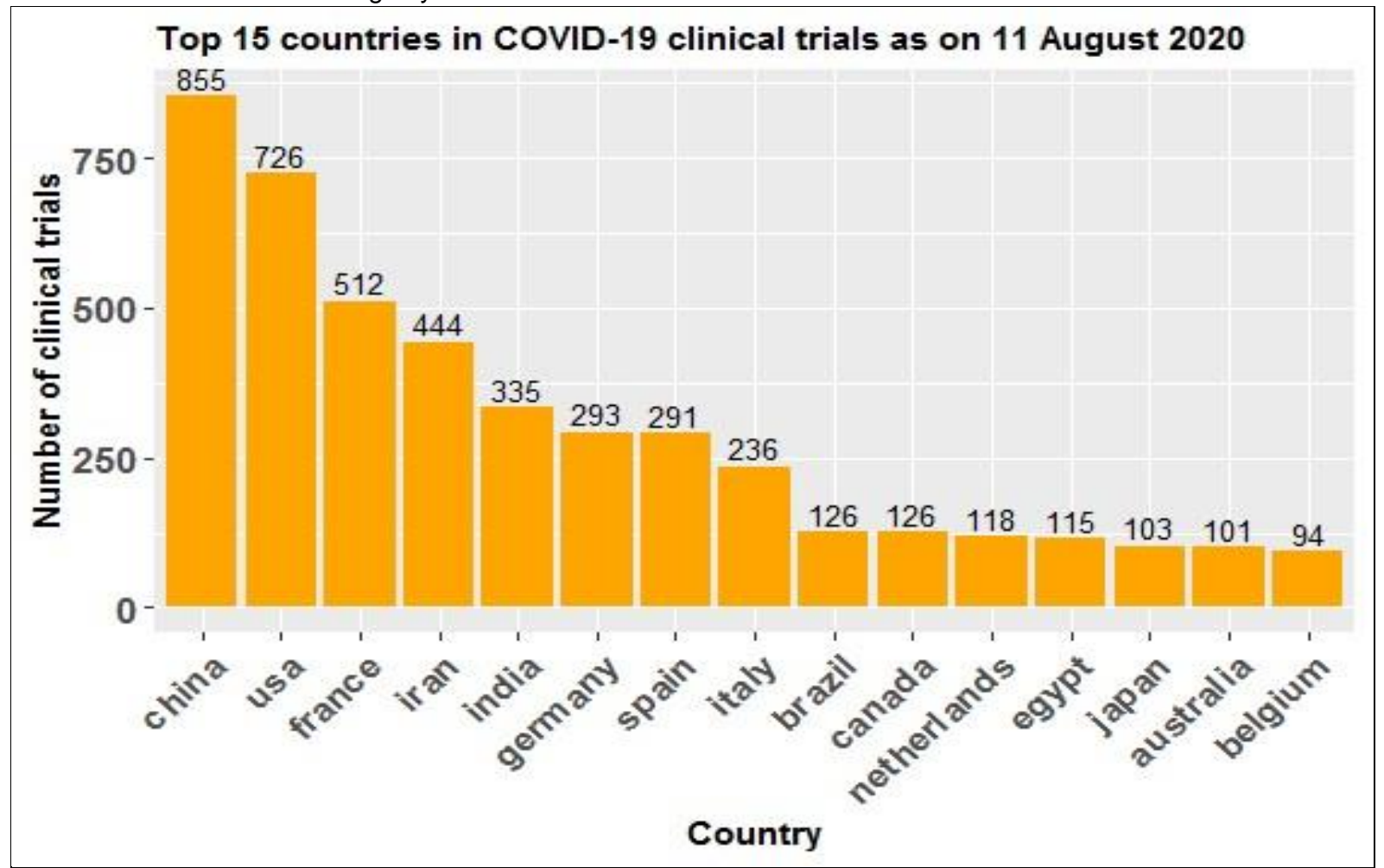

Figure 2. Display the top 15 countries worldwide based on the number of clinical trials registered between 3 February and 11 August 2020 at the International Clinical Trials Registry Platform. 


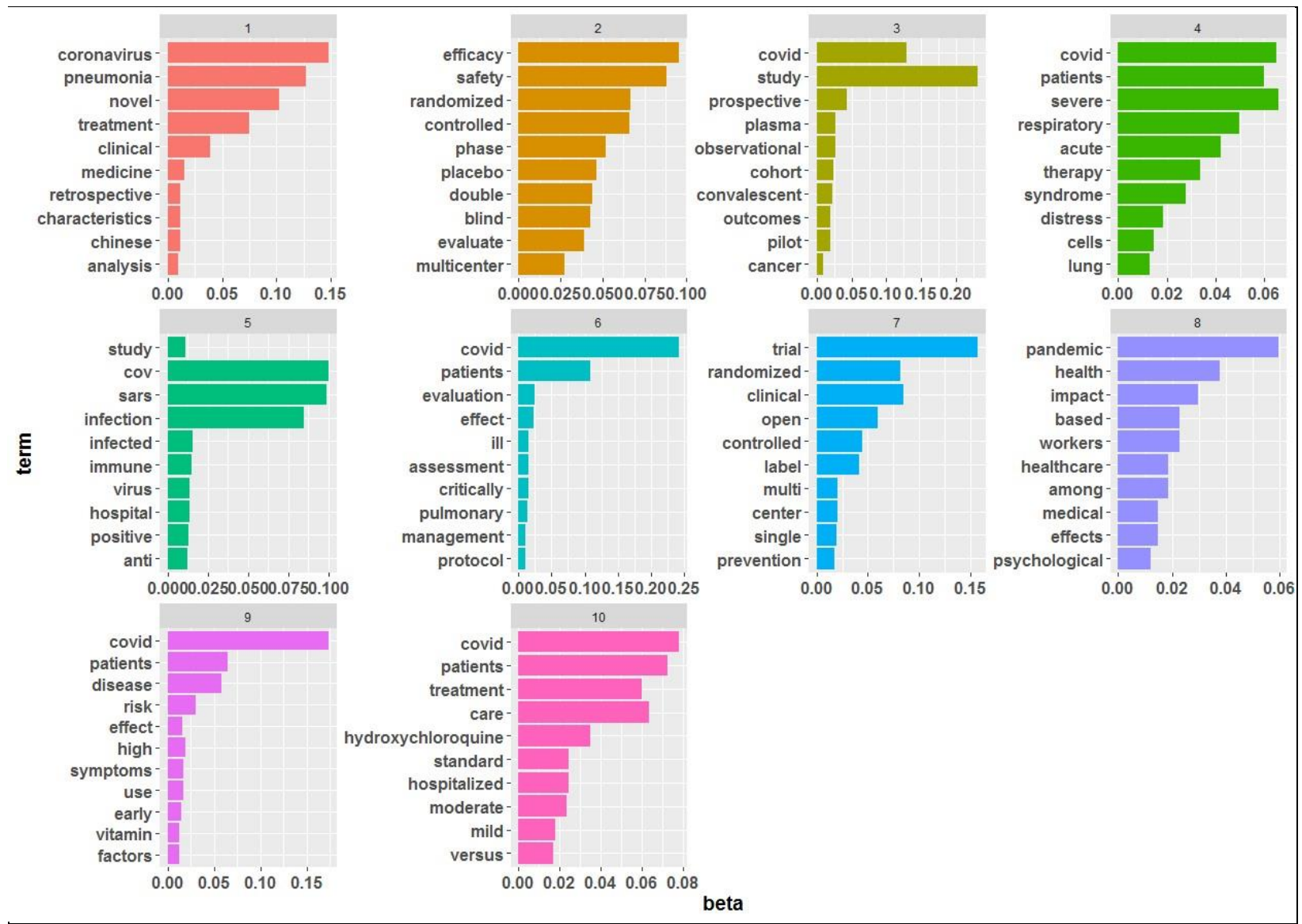

Figure 3. Show the top 10 topics addressed in the reviews and studies of clinical trials related to the advancement of the COVID-19 vaccine created using the LDA topic model on the International Clinical Trials Registry Platform between 3 February and 11 August 2020.

\section{DISCUSSION}

One of goals of this study was to examine whether it was important to calibrate for trials to be included in clinical studies by using freely searchable trial registers instead of massive bibliographic databases such as MEDLINE (U.S. National Library of Medicine). The International Clinical Trials Registry Platform (ICTRP) is a global initiative aimed at making information publicly available on all clinical trials involving humans. In this study, the top 10 topics were addressed in the reviews and studies of clinical trials related to the advancement of the COVID19 vaccine developed using the LDA topic model on the International Clinical Trials Registry Platform between 3 February and 11 August 2020.

This study addressed the clinical trials recorded at ICTRP and showed the reported monthly clinical trials and also the top 15 countries racing to find the COVID-19 vaccine from February 3 to August 11, 2020. Using the collected clinical trials dataset, the LDA topic model was developed to explore emerging trends of vaccine progression.

\section{ACKNOWLEDGMENTS}

Declaration of Conflicting Interests: The authors declare that they have no conflict of interest.

\section{Funding: Not applicable \\ REFERENCES}

1. Huang C, Wang Y, Li X, et al. Clinical features of patients infected with 2019 novel coronavirus in Wuhan, China. Lancet 2020; 395:497-506.

2. World Health Organization. Coronavirus disease (COVID-19) Situation reports. 2020. https://www.who.int/emergencies/diseases/novelcoronavirus-2019/situation-reports (Accessed 17 August 2020) 
3. World Health Organization. News room. 2020. https://www.who.int/news-room/detail/15-07-2020more-than-150-countries-engaged-in-covid-19vaccine-global-access-facility (Accessed 21 August 2020)

4. World Health Organization. Draft landscape of COVID-19 candidate vaccines. 2020. https://www.who.int/publications/m/item/draftlandscape-of-covid-19-candidate-vaccines (Accessed 21 August 2020)

5. Long L, Zeng $X$, Zhang $X$, et al. Short-term outcomes of COVID-19 and risk factors for progression. Eur Respir J 2020; 55: 2000990. doi.org/10.1183/13993003.00990-2020.

6. Xu PP, Tian R, Luo S, et al. Risk factors for adverse clinical outcomes with COVID-19 in China: a multicenter, retrospective, observational study. Theranostics 2020; 10(14): 6372- 6383. doi: $10.7150 /$ thno. 46833

7. Biswas PS et al., A systematic review and Metaanalysis of clinical and laboratory findings of COVID-19, J Microbiol Infect Dis 2020; 10(3):121135.

8. Julie M, et al., Searching ClinicalTrials.gov and the International Clinical Trials Registry Platform to inform systematic reviews: what are the optimal search approaches? J Med Libr Assoc. 2014; 102(3): 177-183. doi: 10.3163/1536-5050.102.3.007

9. Blei DM. Probabilistic topic models. Communications of the ACM 2012; 55(4): 77-84. $10.1145 / 2133806.2133826$ 